\title{
Small-quantity lipid-based nutrient supplements for the prevention of child malnutrition and promotion of healthy development: overview of individual participant data meta-analysis and programmatic implications
}

\author{
Kathryn G Dewey, Christine P Stewart, K Ryan Wessells, Elizabeth L Prado, and Charles D Arnold
}

Institute for Global Nutrition and Department of Nutrition, University of California, Davis, Davis, CA, USA

\begin{abstract}
Small-quantity lipid-based nutrient supplements (SQ-LNSs) were designed to provide multiple micronutrients within a food base that also provides energy, protein, and essential fatty acids, targeted towards preventing malnutrition in vulnerable populations. Previous meta-analyses demonstrated beneficial effects of SQ-LNSs on child growth, anemia, and mortality. To further examine the efficacy and effectiveness of SQ-LNSs, and explore study-level and individuallevel effect modifiers, we conducted an individual participant data meta-analysis of 14 randomized controlled trials of SQ-LNSs provided to children $6-24$ mo of age $(n>37,000)$. We examined growth, development, anemia, and micronutrient status outcomes. Children who received SQ-LNSs had a 12-14\% lower prevalence of stunting, wasting, and underweight; were 16-19\% less likely to score in the lowest decile for language, social-emotional, and motor development; had a $16 \%$ lower prevalence of anemia; and had a $64 \%$ lower prevalence of iron-deficiency anemia compared with control group children. For most outcomes, beneficial effects of SQ-LNSs were evident regardless of study-level characteristics, including region, stunting burden, malaria prevalence, sanitation, water quality, duration of supplementation, frequency of contact, or average reported compliance with SQ-LNSs. For development, the benefits of SQ-LNSs were greater in populations with higher stunting burden, in households with lower socioeconomic status, and among acutely malnourished children. For hemoglobin and iron status, benefits were greater in populations with higher anemia prevalence and among acutely malnourished children, respectively. Thus, targeting based on potential to benefit may be worthwhile for those outcomes. Overall, co-packaging SQ-LNSs with interventions that reduce constraints on response, such as the prevention and control of prenatal and child infections, improving health care access, and promotion of early child development, may lead to greater impact. Policymakers and program planners should consider including SQ-LNSs in strategies to reduce child mortality, stunting, wasting, anemia, iron deficiency, and delayed development. This study was registered at www.crd.yo rk.ac.uk/PROSPERO as CRD42019146592, CRD42020159971, and CRD42020156663. Am J Clin Nutr 2021;114:3S-14S.
\end{abstract}

Keywords: stunting, wasting, undernutrition, anemia, micronutrients, iron status, child development, complementary feeding, nutrient supplements, home fortification

\section{Introduction}

Millions of infants and young children in low- and middleincome countries are vulnerable to undernutrition and impaired neurobehavioral development (1-3). Globally, 21.3\% (144 million) of children under $5 \mathrm{y}$ of age were stunted and $6.9 \%$ (47 million) were wasted in 2019 (2). Deficiencies of micronutrients such as iron, zinc, vitamin A, and vitamin B-12 are widespread, particularly among children under $2 \mathrm{y}$ of age, as a consequence of low micronutrient stores at birth, inadequate dietary intake of bioavailable micronutrients, and increased micronutrient requirements due to infection or malabsorption $(4,5)$. It is

Supported by Bill \& Melinda Gates Foundation grant OPP49817 (to KGD). Published in a supplement to The American Journal of Clinical Nutrition. The Guest Editor for this supplement was Chris Sudfeld, who reports no disclosures. The opinions expressed in this publication are those of the authors and are not attributable to the sponsors or the publisher, Editor, or Editorial Board of The American Journal of Clinical Nutrition. The Supplement Coordinator for the supplement publication was Kathryn Dewey, University of California, Davis, Davis, CA. Kathryn Dewey has received funding from the Bill \& Melinda Gates Foundation and the US Agency for International Development (via the Food and Nutrition Technical Assistance Project) for research on lipid-based nutrient supplements.

Address correspondence to KGD (e-mail: kgdewey@ucdavis.edu).

Abbreviations used: iLiNS, International Lipid-based Nutrient Supplements; IPD, individual participant data; IYCF, infant and young child feeding; LNS, lipid-based nutrient supplement; MNP, multiple micronutrient powder; MUAC, midupper arm circumference; PROMIS, Innovative Approaches for the Prevention of Childhood Malnutrition; RUTF, ready-to-use therapeutic food; SES, socioeconomic status; SQ-LNS, small-quantity lipid-based nutrient supplement; WASH, water, sanitation, and hygiene.

Received January 28, 2021. Accepted for publication August 4, 2021.

First published online September 29, 2021; doi: https://doi.org/10.1093/ ajcn/nqab279. 
estimated that 250 million children under 5 y of age $(43 \%)$ are at risk of not fulfilling their developmental potential (3), and this is linked to inadequate nutrient intake in early life $(6,7)$.

The causes of undernutrition and impaired development are complex and multifactorial (8-13), and thus the impact of interventions that focus solely on improving nutrition may be limited $(10,14)$. Although dietary interventions by themselves may not be sufficient to eliminate these adverse outcomes, they are a necessary element of strategies aimed at children under 2 $\mathrm{y}$ of age, given that inadequate intakes of key nutrients during the complementary feeding period from 6 to 24 mo of age are highly prevalent (8). Improved dietary quality via selection of nutrient-rich complementary foods is the first priority $(15,16)$, but the cost may be prohibitive for low-income households (17, 18). Various types of fortified products have thus been designed to fill nutrient gaps during the period of complementary feeding, such as fortified blended foods, micronutrient powders (MNPs), and lipid-based nutrient supplements (LNSs) (19).

During the past $10 \mathrm{y}$ there has been a rapid expansion of research to evaluate the efficacy and effectiveness of LNSs in various settings, including meta-analyses of effects of prenatal LNSs on birth outcomes (20), and of LNSs for children on multiple outcomes (21) including mortality (22). In the metaanalysis of LNSs given during the period of complementary feeding by Das et al. (21), 17 trials were included, 13 of which provided small-quantity LNSs (SQ-LNSs) in at least 1 arm (the other trials provided larger quantities of LNSs). The authors reported positive results for the prevention of stunting, wasting, and anemia; too few studies were available for child development and micronutrient status outcomes. Although that meta-analysis included some analyses disaggregated by study characteristics, the authors did not conduct analyses stratified by individual-level characteristics.

Since the 2019 meta-analysis by Das et al. (21), additional trials of SQ-LNSs have been completed. To examine the current body of evidence relevant to SQ-LNSs, and explore reasons for heterogeneity in results, we conducted an individual participant data (IPD) meta-analysis of randomized controlled trials of SQLNSs provided to infants and young children 6 to 24 mo of age. Our objectives were to 1) generate pooled estimates of the effect of SQ-LNSs on outcomes in 3 different domains-a) growth, b) development, and c) anemia and micronutrient status - and 2) identify study-level and individual-level modifiers of the effect of SQ-LNSs on those outcomes. Identification of subgroups of infants and young children who experience greater benefits from SQ-LNSs, or are more likely to respond to the intervention, may be useful in informing the development of public health programs and policies (14). This overview provides a brief history of the development and evaluation of SQ-LNSs, a synopsis of the methods of the IPD meta-analyses and the trials included, and a synthesis of the results including discussion of the programmatic and policy implications. Three other papers in this supplement issue report the detailed methods and results for each of the 3 outcome domains (23-25).

\section{Development and Evaluation of SQ-LNSs}

In the late 1990s, the first ready-to-use therapeutic food (RUTF) for treating severe malnutrition (Plumpy'nut ${ }^{\circledR}$, Nutriset, France) was developed, based on embedding micronutrients in a fat-based matrix. This technology allows the product to have a low water activity, which is critical because it inhibits the growth of harmful microorganisms without refrigeration, permitting treatment in outpatient settings. Community-based management of severe malnutrition using RUTF subsequently became widespread. While this revolutionized strategies for the treatment of malnutrition, there were limited options with regard to effective strategies for the prevention of malnutrition. To address the latter need, SQ-LNSs were developed based on the same type of food-based matrix used for RUTF (including vegetable oil, peanut paste, and milk powder), but using a much smaller quantity of food, typically about 4 teaspoons ( $100-120 \mathrm{kcal})$ per day (26). The food base provides energy, protein, and essential fatty acids; together with the multiple micronutrients added via fortification, the combination addresses multiple potential nutritional deficiencies.

The target quantity of food in SQ-LNSs designed for infants and young children was small for several reasons. First, it is important to avoid displacing breast milk and locally available nutrient-rich foods. The energy needed from complementary foods, assuming average breast-milk intakes, is only $\sim 200 \mathrm{kcal}$ at 6-8 mo, $\sim 300$ at $9-11 \mathrm{mo}$, and $\sim 550 \mathrm{kcal}$ at $12-23 \mathrm{mo}$ of age. The proportions of these energy needs provided by SQ-LNSs are approximately one-half at 6-8 mo, one-third at 9-11 mo, and onefifth at 12-23 mo, leaving room for other complementary foods in the diet. Second, the small quantity of the daily ration of SQLNSs makes it likely that the child can consume the entire ration in $1 \mathrm{~d}$, thereby receiving the intended doses of the micronutrients and essential fatty acids. With a larger quantity, such as mediumquantity LNSs [typically $250-500 \mathrm{kcal} / \mathrm{d}$ (26)], a substantial amount may be left unconsumed (27), particularly by infants 612 mo of age. Third, the daily ration of SQ-LNSs can easily be mixed with other foods [and thus SQ-LNSs are considered a type of home fortification product (28)] or consumed as is, allowing for flexibility in feeding practices. Last, the cost of production and transport of LNSs, and the feasibility of distribution via platforms such as community health workers, is related to the quantity per recipient, so SQ-LNSs are a lower-cost option than other fortified products designed for a larger daily ration.

The first trials evaluating SQ-LNSs were published in 20072008 , and results were promising with regard to the prevention of linear growth faltering in Ghana (29) and of severe stunting in Malawi (30). Subsequently, the International Lipid-based Nutrient Supplements (iLiNS) Project (https://ilins.ucdavis.edu/) was funded by the Bill \& Melinda Gates Foundation to develop several modified formulations of SQ-LNSs (including a version for pregnant and lactating women), conduct large randomized controlled efficacy trials in 3 countries in Africa, conduct socioeconomic studies of SQ-LNSs, and coordinate efforts among stakeholders. For the latter objective, the iLiNS Project facilitated the LNS Research Network between 2009 and 2015 to promote the exchange of information and experience among researchers and practitioners. These efforts accelerated research on SQ-LNSs in a variety of contexts by numerous investigators, both within and outside of the iLiNS Project. This work included studies of acceptability and adherence, trials assessing efficacy and effectiveness for improving a range of different outcomes among infants and young children, as well as pregnant and lactating women, and studies on costs and willingness to pay for SQ-LNSs. In addition, several trials evaluated SQ-LNS interventions with and without co-interventions aimed at improving water, sanitation, and 
hygiene (WASH), such as the WASH-Benefits trials in Bangladesh (2012-2015) (31) and Kenya (2012-2016) (32) and the SHINE trial in Zimbabwe (2013-2017) (33, 34). Two trials tested the impact of linking prevention with treatment (called Innovative Approaches for the Prevention of Childhood Malnutrition, or PROMIS), by incorporating SQLNS distribution into programs that included screening for acute malnutrition in the community (PROMIS-Mali, 2015-2017) (35) or health clinic (PROMIS-Burkina Faso, 2015-2017) (36).

Researchers designing trials to evaluate SQ-LNSs have recognized that provision of supplements needs to be accompanied by appropriate messages. These include not just information about the use and storage of SQ-LNSs but also messages to emphasize recommended infant and young child feeding (IYCF) practices. These messages have typically included the promotion of breastfeeding, introduction of complementary foods at 6 mo of age, and recommendations regarding dietary diversity and feeding nutrient-rich complementary foods. These messages have usually been provided to both intervention and control groups, to reinforce the normal IYCF messages already promoted in the study location. Some studies have gone further by providing expanded social and behavior change communication on IYCF. Thus, provision of SQ-LNSs generally occurs within an overall context to improve IYCF, not as an isolated intervention solely focused on delivering a product.

\section{SQ-LNS IPD Meta-analysis}

\section{Overview of methods}

The IPD meta-analysis presented in the accompanying articles is based on pooled data from 14 randomized controlled trials of SQ-LNSs. This work followed best practices for pre-registration, transparency, and reproducibility, with protocols and statistical analysis plans posted online (osf.io/ymsfu and at PROSPERO CRD42019146592, CRD42020159971, and CRD42020156663) (37-40). The trials were identified beginning with those published in a recent Cochrane Review (21), and supplemented with additional studies identified through a systematic review of studies published through September 2019. Investigators who led trials meeting the eligibility criteria shown in Text Box 1 were invited to contribute data and participate in the analysis.

The main comparison of interest was the provision of SQ-LNSs ( $<\sim 125 \mathrm{kcal} / \mathrm{d}$, with or without co-interventions) compared with provision of no intervention or an intervention without any type of LNS or other child supplement. For trials with multiple relevant SQ-LNS interventions (e.g., varying dosages or formulations of SQ-LNSs in different arms), combined provision of child SQ-LNSs with provision of maternal LNSs, or inclusion of other nonnutritional interventions (i.e., WASH), all arms that provided child SQ-LNSs were combined into 1 group. All nonLNS arms (i.e., no LNSs for mother or child) were combined into a single comparator ("control") group for each trial (excluding intervention arms that received non-LNS child supplementation, e.g., MNP, fortified-blended food). For trials that provided both maternal and child LNSs, we conducted analyses both with ("all-trials analysis") and without ("child-LNS-only") the maternal LNS arms. The main effects did not differ between these 2 analyses by more than $20 \%$ for continuous outcomes or by 0.05 for prevalence ratios, so the results of the all-trials analyses are presented as the principal findings.
We conducted several prespecified sensitivity analyses: 1) separate comparisons within multicomponent intervention trials, such that the SQ-LNS to no-SQ-LNS comparisons were conducted separately between pairs of arms with the same nonnutrition components (e.g., SQ-LNS + WASH vs. WASH; SQLNS vs. control); 2) exclusion of passive control arms (i.e., control group participants received no intervention and had no contact with project staff between baseline and endline); and 3) exclusion of intervention arms with SQ-LNS formulations that did not include both milk and peanut.

Three types of statistical analyses were conducted to separately investigate 1) full sample treatment effects, 2) effect modification by study-level characteristics, and 3) effect modification by individual-level characteristics. For all 3 sets of analyses, we used a 2-stage approach following a complete-case intention-totreat framework. We used bivariate meta-regression modeling to examine effect modification by study-level characteristics. We modeled effect modification by individual-level characteristics within each study, and then pooled the estimates. Potential effect modifiers examined are shown in Table $\mathbf{1}$.

\section{TEXT BOX 1}

Eligibility criteria for the SQ-LNS IPD analyses

Inclusion criteria

- Randomized controlled trial

- Conducted in low- or middle-income country

- SQ-LNSs ( $<\sim 125 \mathrm{kcal} / \mathrm{d})$ provided to the intervention group for at least 3 mo between 6 and 24 mo of age

- At least 1 trial group did not receive SQ-LNSs or other type of child supplementation

- Longitudinal follow-up of each child, or repeated cross-sectional data collection

- Individual children eligible for IPD analysis if:

- Age at baseline allowed receipt of intervention (supplementation or control group components) for at least 3 mo between 6 and 24 mo of age

- For anemia and micronutrient status outcomes, blood samples were collected during the supplementation period or within 3 mo after the study-defined end of supplementation

Exclusion criteria

- LNS was used for treatment, not prevention, of malnutrition (i.e. only children with severe or moderate malnutrition were eligible for the study)

- Conducted in a hospitalized population or among children with a pre-existing disease

- SQ-LNS provision was combined with additional supplemental food or nutrients for the child within a single arm (e.g., SQ-LNS + food rations vs. control), and there was no appropriate comparison group (e.g., food rations alone) that would allow separation of the SQ-LNS effect from effects of the other food or nutrients provided

\section{Trials included}

We identified 15 trials that met our inclusion criteria, 14 of which provided individual participant data and were included in 
TABLE 1 Potential effect modifiers considered within each outcome domain ${ }^{1}$

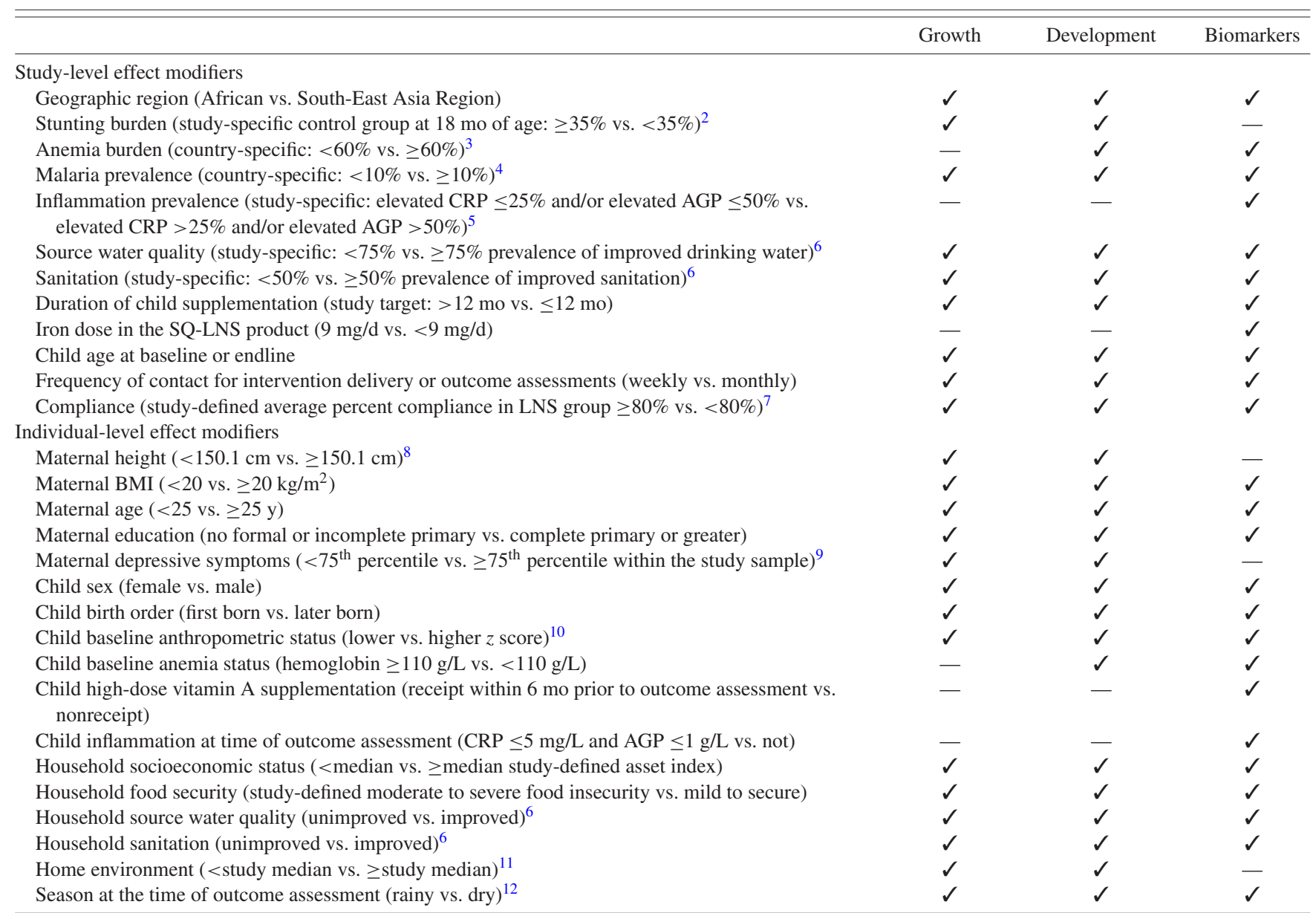

${ }^{1}$ AGP, $\alpha 1$-acid glycoprotein; CRP, C-reactive protein; HCZ, head circumference-for-age $z$ score; LAZ, length-for-age $z$ score; LNS, lipid-based nutrient supplement; MUAC, midupper arm circumference; MUACZ, midupper arm circumference $z$ score; SQ-LNS, small-quantity lipid-based nutrient supplement; WASH, water, sanitation, and hygiene; WAZ, weight-for-age $z$ score; WLZ, weight-for-length $z$ score.

${ }^{2}$ Based on 18-mo data because baseline data not available for all trials; cutoff chosen at approximately the median across trials.

${ }^{3}$ Country-specific prevalence of anemia among children 6-59 mo, based on national surveys closest in time to the study; cutoff chosen based on the median across trials.

${ }^{4}$ Country-specific prevalence of malaria closest in time to the study, based on World Malaria Report 2018 (66); cutoff chosen based on the median across trials.

${ }^{5}$ Elevated CRP defined as $>5 \mathrm{mg} / \mathrm{L}$, elevated AGP defined as $>1 \mathrm{~g} / \mathrm{L}$; cutoff chosen based on the median across trials.

${ }^{6}$ Improved drinking water and sanitation quality defined using WHO/UNICEF Joint Monitoring Program definitions (67, 68); based on baseline data, excluding arms that received WASH interventions; cutoff chosen at approximately the median across trials.

${ }^{7}$ Study-specific, as reported based on a study-defined indicator; cutoff chosen based on the median across trials.

${ }^{8}$ Cutoff based on -2 SDs for height at 19 y of age at https://www.who.int/growthref/hfa_girls_5_19years_z.pdf?ua=1.

${ }^{9}$ Study-specific; cutoff chosen to reflect top quartile for risk of depression.

${ }^{10}$ For the growth domain, this was defined as LAZ $<$ vs. $\geq-1$ when LAZ or stunting was the outcome; WLZ $<$ vs. $\geq 0$ when WLZ, wasting, or acute malnutrition was the outcome; MUACZ $<$ vs. $\geq 0$ when MUACZ or low MUAC was the outcome; WAZ $<$ vs. $\geq-1$ when WAZ or underweight was the outcome; $\mathrm{HCZ}<$ vs. $\geq-1$ when $\mathrm{HCZ}$ or small head size was the outcome. For the development domain, this was defined as LAZ $<$ vs. $\geq-2$ for all outcomes. For the anemia and micronutrient status domain this was defined as WLZ $<-2$ or MUAC $<125 \mathrm{~mm}$ vs. WLZ $\geq-2$ and MUAC $\geq 125 \mathrm{~mm}$; if MUAC not measured, WLZ $<$ vs. $\geq-2$.

${ }^{11}$ As measured by the Family Care Indicators, Home Observation for the Measurement of the Environment Inventory, or other similar tools.

${ }^{12}$ Based on average rainfall during the month of outcome assessment and 2 mo prior.

all analyses (Table 2) (29, 31-36, 41-48). Investigators for 1 trial were unable to participate (49). One trial was designed a priori to present results separately for HIV-exposed and HIV-unexposed children and is presented as 2 separate comparisons in all analyses $(33,34)$. Similarly, the 2 PROMIS trials in Burkina Faso and Mali each included an independent longitudinal cohort and repeated (at baseline and endline) cross-sectional samples, so the longitudinal and cross-sectional results are presented as separate comparisons for each trial $(35,36)$.

The 14 trials in these analyses were conducted in sub-Saharan Africa (10 trials in 7 countries), Bangladesh ( 3 trials), and Haiti (1 trial), and included a total of 37,066 infants and young children. Most trials began child supplementation with SQ-LNSs at 6 mo of age and the intended duration ranged from 6 to 18 mo of 
TABLE 2 Characteristics of the trials included in the SQ-LNS IPD meta-analyses ${ }^{1}$

\begin{tabular}{|c|c|c|c|c|c|c|}
\hline \multirow[b]{2}{*}{ Country } & \multirow[b]{2}{*}{ Study (reference) } & \multirow[b]{2}{*}{ Trial name } & \multicolumn{2}{|c|}{ Infant SQ-LNSs } & \multirow[b]{2}{*}{ Maternal LNSs } & \multirow[b]{2}{*}{ Participants } \\
\hline & & & Age at start, mo & Duration, mo & & \\
\hline Bangladesh & Christian 2015 (41) & JiVitA-4 & 6 & 12 & $\mathrm{~N}$ & 4218 \\
\hline Bangladesh & Dewey 2017 (42) & RDNS & 6 & 18 & $\mathrm{Y} / \mathrm{N}$ & 2478 \\
\hline Bangladesh & Luby 2018 (31) & $\begin{array}{c}\text { WASH-Benefits- } \\
\text { Bangladesh }\end{array}$ & 6 & 18 & $\mathrm{~N}$ & 4633 \\
\hline Burkina Faso & Hess 2015 (43) & iLiNS-ZINC & 9 & 9 & $\mathrm{~N}$ & 2626 \\
\hline Burkina Faso & Becquey 2019 (36) & $\begin{array}{l}\text { PROMIS-Burkina } \\
\text { Faso }\end{array}$ & 6 & 12 & $\mathrm{~N}$ & 2651 \\
\hline Ghana & Adu-Afarwuah 2007 (29) & & 6 & 6 & $\mathrm{~N}$ & 194 \\
\hline Ghana & Adu-Afarwuah 2016 (44) & iLiNS-DYAD-Ghana & 6 & 12 & $\mathrm{Y}$ & 1040 \\
\hline Haiti & Iannotti $2014(45)$ & & $6-11$ & $3-6$ & $\mathrm{~N}$ & 300 \\
\hline Kenya & Null 2018 (32) & $\begin{array}{l}\text { WASH-Benefits- } \\
\text { Kenya }\end{array}$ & 6 & 18 & $\mathrm{~N}$ & 6649 \\
\hline Madagascar & Galasso 2019 (46) & MAHAY & $6-11$ & $6-12$ & $\mathrm{Y} / \mathrm{N}$ & 3390 \\
\hline Malawi & Ashorn 2015 (47) & $\begin{array}{l}\text { iLiNS-DYAD- } \\
\text { Malawi }\end{array}$ & 6 & 12 & $\mathrm{Y}$ & 664 \\
\hline Malawi & Maleta 2015 (48) & iLiNS-DOSE & 6 & 12 & $\mathrm{~N}$ & 943 \\
\hline Mali & Huybregts 2019 (35) & PROMIS-Mali & 6 & 18 & $\mathrm{~N}$ & 2937 \\
\hline Zimbabwe & Humphrey 2019 (33) & SHINE & 6 & 12 & $\mathrm{~N}$ & 3676 \\
\hline Zimbabwe & Prendergast 2019 (34) & $\begin{array}{l}\text { SHINE } \\
\quad(\text { HIV-exposed) }\end{array}$ & 6 & 12 & $\mathrm{~N}$ & 667 \\
\hline
\end{tabular}

${ }^{1}$ IPD, individual participant data; LNS, lipid-based nutrient supplement; N, no; PROMIS, Innovative Approaches for the Prevention of Childhood Malnutrition; RDNS, Rang-Din Nutrition Study; SHINE, Sanitation, Hygiene, Infant Nutrition Efficacy Project; SQ-LNS, small-quantity lipid-based nutrient supplement; Y, yes; Y/N indicates that the trial had at least one arm with maternal LNSs + child LNSs and at least one arm with child LNSs only.

supplementation; 4 trials included intervention arms that also provided SQ-LNSs to mothers during pregnancy and the first 6 mo postpartum $(42,44,46,47)$. All trials provided a peanut- and milk-based SQ-LNS in at least 1 of the arms.

Six trials were conducted within existing community-based or clinic-based programs (33-36, 42, 45, 46); in the other trials, all activities were conducted by research teams. Seven trials provided minimal messaging on IYCF other than reinforcing the normal IYCF messages already promoted in that setting $(29,42-$ $45,47,48)$, and 7 trials provided expanded social and behavioral change communication on IYCF that went beyond the usual messaging, either in just the SQ-LNS intervention arms (31-34, $36)$ or in all arms including the non-SQ-LNS control arm (35, 41, 46). Three trials included arms with WASH interventions (31-34). Most trials included an active control arm (i.e., similar contact frequency as for intervention arms) but 3 included only a passive control arm $(29,31,43)$.

The 14 study sites were highly diverse in terms of study-level characteristics, including stunting burden, malaria prevalence, water quality, sanitation, and aspects of study design such as duration of supplementation, frequency of contact, and average compliance with SQ-LNSs. There was also wide variation within and between studies in maternal, child, and household characteristics. This provided heterogeneity for exploration of potential effect modifiers.

\section{Synthesis of results and programmatic implications}

\section{Main effects of SQ-LNSS.}

Overall, when combining data from all of the trials, we found significant effects of SQ-LNSs across all 3 outcome domains
(Table 3). Children who received SQ-LNSs had a 12-14\% lower prevalence of stunting, wasting, and underweight (23); were 16-19\% less likely to score in the lowest decile for language, social-emotional, and motor development (24); and had a $16 \%$ lower prevalence of anemia and $64 \%$ lower prevalence of irondeficiency anemia (25) compared with control children who did not receive SQ-LNSs. These findings add to those of a recently published meta-analysis of many of these same trials reporting a $27 \%$ lower risk of mortality between 6 and 24 mo of age (22).

This IPD meta-analysis includes nearly 3 times as many participants as the meta-analysis by Das et al. (21), even though we restricted the analysis to trials that provided SQ-LNSs. Our new estimates for wasting and underweight are similar to those of Das et al., but the new estimate for stunting (12\% relative reduction) is larger than previously reported (7\% reduction). For child development outcomes, Das et al. provided a narrative review of effects but were not able to generate pooled estimates. With regard to anemia, Das et al. reported a relative reduction of $21 \%$; our $16 \%$ reduction is somewhat lower than that, but we defined anemia as hemoglobin $<110 \mathrm{~g} / \mathrm{L}$, whereas Das et al. examined anemia as defined by trialists. In addition, we report 56-64\% reductions in iron deficiency and iron-deficiency anemia, which were not reported in the meta-analysis by Das et al. The larger reduction in the prevalence of iron-deficiency anemia as compared with all-cause anemia reflects the complex etiology of anemia; supplementation is likely to prevent only the fraction of anemia attributable to nutritional causes.

The overall effects of SQ-LNSs on the growth and development outcomes were modest. However, the effects were generally more consistent, and for some outcomes more substantial, compared with other nutrition interventions for children under 2 y of age, such as nutrition education, micronutrient supplementation 
TABLE 3 Relative reductions in adverse outcomes in meta-analyses of intervention trials providing SQ-LNSs to children 6-24 mo of age ${ }^{1}$

\begin{tabular}{|c|c|}
\hline & $\begin{array}{l}\text { Relative reduction } \\
\quad(95 \% \mathrm{CI}), \%\end{array}$ \\
\hline \multicolumn{2}{|l|}{ Growth outcomes } \\
\hline Stunting (LAZ < -2 SDs) & $12(9,15)$ \\
\hline Wasting (WLZ $<-2$ SDs) & $14(7,20)$ \\
\hline Underweight (WAZ $<-2$ SDs) & $13(9,17)$ \\
\hline Acute malnutrition (WLZ $<-2$ SDs or MUAC $<125 \mathrm{~mm}$ ) & $14(7,20)$ \\
\hline Low MUAC (MUACZ < -2 SDs or MUAC < $125 \mathrm{~mm}$ ) & $18(11,25)$ \\
\hline Small head circumference $(\mathrm{HCZ}<-2 \mathrm{SDs})$ & $9(5,14)$ \\
\hline \multicolumn{2}{|l|}{ Development outcomes ${ }^{2}$} \\
\hline Low language development score & $16(8,24)$ \\
\hline Low motor development score & $16(8,24)$ \\
\hline Low social-emotional development score & $19(11,26)$ \\
\hline \multicolumn{2}{|l|}{ Anemia and micronutrient status outcomes } \\
\hline Anemia $(\mathrm{Hb}<110 \mathrm{~g} / \mathrm{L})$ & $16(13,19)$ \\
\hline Moderate-severe anemia $(\mathrm{Hb}<100 \mathrm{~g} / \mathrm{L})$ & $28(24,32)$ \\
\hline Iron deficiency (Ferritin $<12 \mu \mathrm{g} / \mathrm{L}$ ) & $56(50,61)$ \\
\hline Iron deficiency anemia $(\mathrm{Hb}<110 \mathrm{~g} / \mathrm{L}$ and ferritin $<12 \mu \mathrm{g} / \mathrm{L})$ & $64(56,70)$ \\
\hline Vitamin A deficiency $(\mathrm{RBP}<0.70 \mu \mathrm{mol} / \mathrm{L})$ & $56(30,73)$ \\
\hline Mortality & $27(11,41)$ \\
\hline \multicolumn{2}{|c|}{$\begin{array}{l}{ }^{1} \text { Based on Dewey et al. [(23), growth outcomes], Prado et al. [(24), development outcomes], Wessells et al. [(25), } \\
\text { anemia and micronutrient status outcomes], and Stewart et al. [(22), mortality]. Hb, hemoglobin; HCZ, head } \\
\text { circumference-for-age } z \text { score; LAZ, length-for-age } z \text { score; MUAC, midupper arm circumference; MUACZ, } \\
\text { midupper arm circumference } z \text { score; RBP, retinol binding protein; SQ-LNS, small-quantity lipid-based nutrient } \\
\text { supplement; WAZ, weight-for-age } z \text { score; WLZ, weight-for-length } z \text { score. }\end{array}$} \\
\hline
\end{tabular}

or fortification, and fortified blended foods (50-54). Many studies have examined the impacts of behavior change interventions focused on improving complementary feeding practices. A 2018 Cochrane review concluded that such interventions are effective at improving reported feeding practices, but there was insufficient evidence to draw conclusions with respect to effects on growth, development, anemia, or micronutrient status (51). Within our IPD analysis, 3 trials had direct comparisons of LNS + IYCF behavior change compared with IYCF behavior change alone: the JiVitA-4 trial in Bangladesh (41), the PROMIS study in Mali (35), and the Mahay study in Madagascar (46). In 2 of these 3 trials (JiVitA-4 and PROMIS-Mali), the children in the LNS intervention arm had improved growth, hemoglobin, and motor development scores compared with children in the IYCF-only arm.

Effects of SQ-LNSs on anemia and iron deficiency were similar to those reported in a recent meta-analysis of MNP [18\% reduction in anemia and 53\% reduction in iron deficiency (55)]. However, that review demonstrated no effects of MNP on child growth and there was insufficient evidence to evaluate effects on child development or mortality.

Compared with interventions promoting responsive care and provision of learning opportunities for young children, effects of SQ-LNSs on mean scores for child development were substantially smaller: pooled effect sizes for the former ranged from 0.39 to $0.49 \mathrm{SD}$ higher scores for language, socialemotional, and motor development $(56,57)$ compared with 0.07 to 0.08 SD higher scores for those domains in the SQ-LNS IPD analysis. However, the former trials did not report estimates for the prevalence of children scoring below a given cutoff in each domain, so we cannot directly compare results with the impact of SQ-LNSs on the percentage of children scoring in the lowest decile (relative reductions of 16-19\%). Many of the responsive care and learning opportunities studies did not measure indicators of child growth; however, among those that did, there was little evidence of effect (56). Thus, the 2 types of interventions may be complementary.

\section{Effect modification.}

Each of the accompanying papers presents detailed information on the effect modification results. For most of the outcome domains, effect modification was examined in 3 ways: as the mean difference in the continuous outcome (e.g., lengthfor-age $\mathrm{z}$ score) between SQ-LNS and control groups, as the prevalence ratio between the 2 groups for a binary outcome (e.g., the relative risk of stunting), and as the difference in absolute prevalence between the 2 groups for a binary outcome (e.g., the percentage point difference in prevalence of stunting). We considered all 3 types of outcomes together and in reference to 3 different theoretical scenarios: potential to benefit, potential to respond, and cutoff effects.

Potential to benefit. One possibility is that certain subgroups of children may be more likely to benefit from the SQ-LNS intervention, perhaps due to greater nutritional deficits at baseline (14). This is illustrated in Figure 1A. The children with greater nutritional or developmental deficits in subgroup a-1 are shown 


\section{A Potential to benefit}

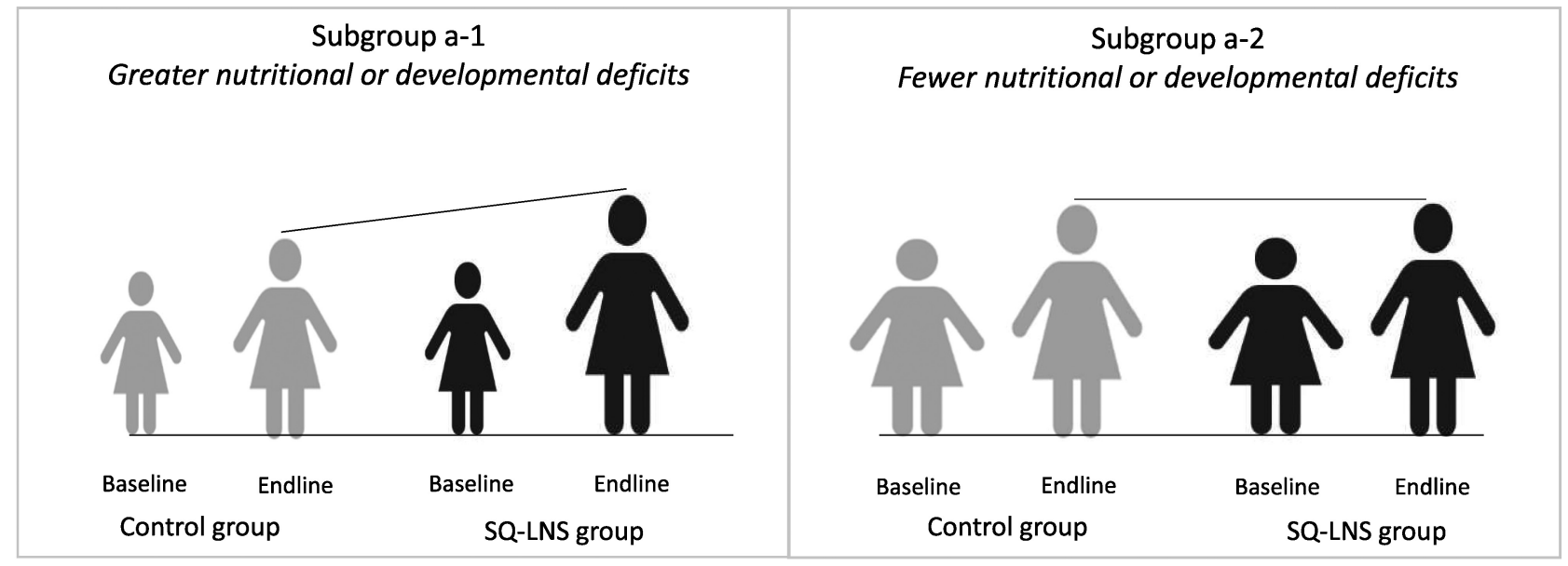

\section{B Potential to respond}

Subgroup b-1
Fewer constraints on response

FIGURE 1 Illustration of the concepts of potential to benefit and potential to respond. (A) Subgroup a-1 has greater nutritional deficits, here illustrated as being slightly smaller and thinner than subgroup a-2, and therefore has a greater potential to benefit from a nutritional intervention. (B) Subgroup b-1 has a greater potential to respond to a nutritional intervention, due to fewer constraints, as compared with subgroup b-2. SQ-LNS, small-quantity lipid-based nutrient supplements.

here as being slightly smaller and thinner compared with the children in subgroup a-2. When SQ-LNS is provided, the children in subgroup a-1 may benefit more. Our interpretation in these cases is that there were greater benefits to those who needed it most.

Potential to respond. Another possibility is that certain subgroups may differ in their potential to respond to the intervention (14) as a result of constraints such as infection or inflammation, caregiver time or resources, or chronic stress. In Figure 1B, subgroup b-2 has greater constraints, whereas subgroup b-1 has no such constraints. In this case, we might expect to see a greater response to supplementation in subgroup b-1.

Cutoff effects. Effect modification results may differ as an artifact of where the continuous outcome distribution falls with respect to the cutoff value for a binary variable. In Figure 2, subgroup B has a higher proportion of observations clustered near the cutoff value. Assuming similar shifts in the means of the distributions within both subgroups in response to the intervention, a very different proportion of children cross the cutoff value threshold. In this example, a greater proportion of children in subgroup B would cross that threshold as compared with subgroup A. Thus, there may be statistically significant effect modification for a binary outcome measure but not for the corresponding continuous outcome measure. In these situations, if there is no significant effect modification for the continuous outcome, we interpret the results as evidence that both subgroups benefit and respond similarly. For this reason, we have not highlighted these examples for further discussion here, but they are described in the accompanying papers (23-25).

For growth and development outcomes, most of the study-level characteristics and aspects of study design did not significantly modify the effects of SQ-LNSs; the exceptions were stunting and anemia burdens, which were related to the impact of SQ-LNSs 


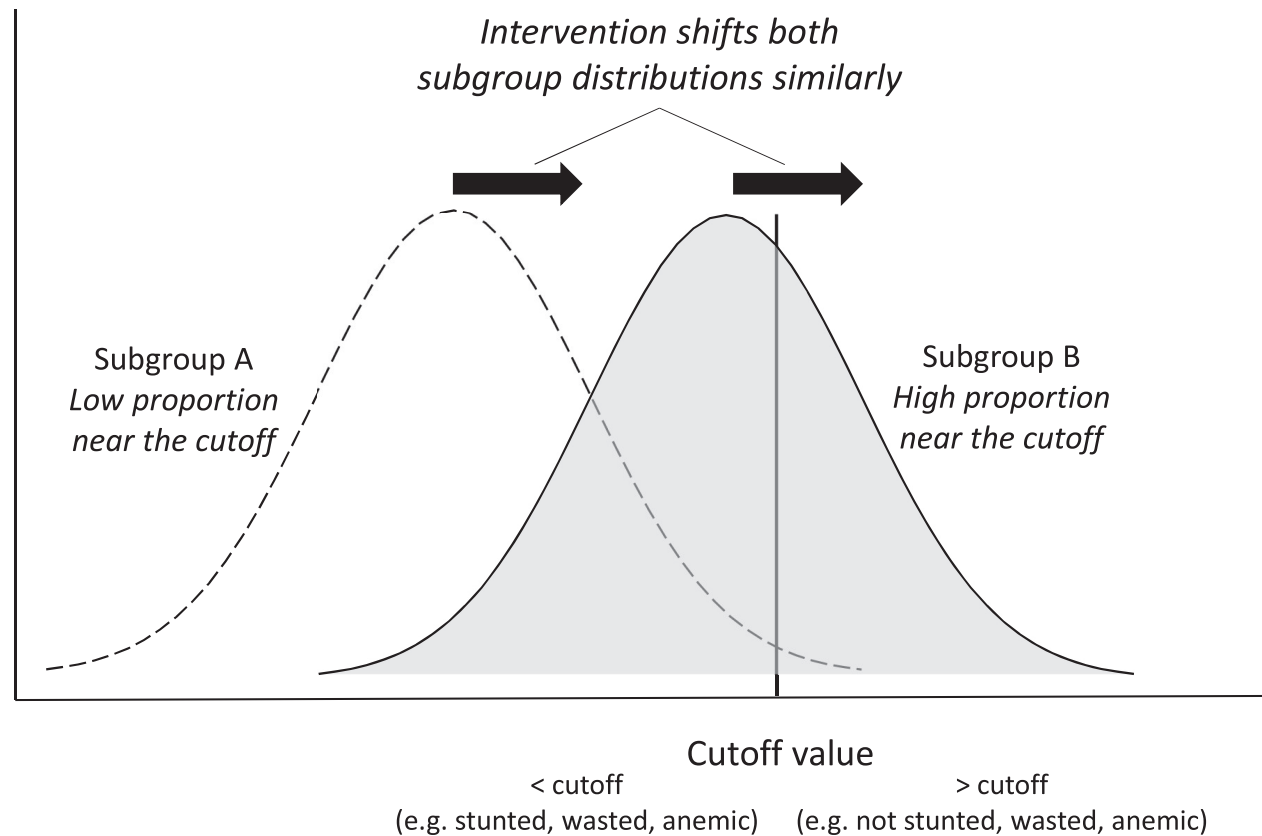

FIGURE 2 Illustration of the cutoff effect. The intervention shifts both subgroup distributions similarly, resulting in a higher proportion of the individuals in subgroup B crossing the cutoff value threshold. SQ-LNS, small-quantity lipid-based nutrient supplements.

on child development outcomes. For anemia and iron deficiency, several study characteristics (region, anemia burden, malaria prevalence, and prevalence of inflammation) and aspects of study design (duration of supplementation, iron dose in SQ-LNSs, and average compliance with SQ-LNSs) appeared to modify the effects of SQ-LNSs. For all outcomes, there was significant effect modification by individual-level characteristics, but the characteristics that appeared to be important varied considerably depending on the type of outcome. In the paragraphs that follow, we discuss effect modifiers that were common across multiple outcome domains or that might have programmatic implications, using the concepts of potential to benefit and potential to respond described above to interpret the findings.

For 2 characteristics, child-level acute malnutrition and country-level anemia burden, the results suggested that populations with greater nutritional deficits may have greater potential to benefit from supplementation. Among children acutely malnourished at baseline, plasma ferritin concentration increased by $85 \%$ and language, social-emotional, and motor development scores increased by approximately $0.3 \mathrm{SDs}$ in the SQ-LNS compared with control groups. In contrast, among children not acutely malnourished, there was a smaller, although still significant, impact on plasma ferritin ( $47 \%$ increase) and an increase of only $\sim 0.1 \mathrm{SD}$ in developmental scores. Similarly, in sites with a higher anemia burden, there were larger differences in mean hemoglobin $(+4.9$ in SQ-LNS vs. control groups in highanemia-burden sites compared with $+2.5 \mathrm{~g} / \mathrm{L}$ in low-anemiaburden sites), and larger reductions in the prevalence of moderateto-severe anemia ( -13 vs. -4 percentage points) and lowest decile of motor scores ( $25 \%$ vs. $12 \%)$.

Effect modification by 2 other characteristics, household socioeconomic status (SES) and study-level stunting burden, suggested that children experiencing greater levels of poverty benefitted more with respect to the effect of SQ-LNSs on child development. Such children may be at greater risk of developmental delay (24) and therefore may have a greater potential to benefit developmentally from supplementation. The mean effects of SQ-LNSs on language, social-emotional, and motor development scores in low-SES households were +0.06 to +0.12 SDs, compared with little to no effects among children in higher SES households. Similarly, in sites with a high stunting burden, language, social-emotional, and motor development scores were +0.08 to +0.13 SDs higher in the SQ-LNS compared with control groups; in contrast, there were no effects on these outcomes in settings with a low stunting burden. While these differences were apparent for the development outcomes, SES and stunting burden generally did not significantly modify the effects of SQ-LNSs on growth, anemia, or iron status outcomes.

Child sex was an effect modifier of the effect of SQ-LNSs on growth and anemia, with stronger effects among girls than boys. Among girls, SQ-LNSs reduced the prevalence of stunting by $16 \%$ (vs. $9 \%$ among boys), wasting by $21 \%$ (vs. 10\%), low midupper arm circumference (MUAC) by $27 \%$ (vs. 7\%), small head size by $15 \%$ (vs. $4 \%$ ), and anemia by $18 \%$ (vs. 13\%), although the latter difference was likely due to the cutoff effect. Girls had higher mean anthropometric $z$ scores and hemoglobin concentrations than boys, which suggests that they did not have a greater potential to benefit from the supplementation. Rather, they may have had a greater potential to respond. Boys are at greater risk of morbidity and mortality in early life and may be more vulnerable to environmental stressors (58), which could constrain their response to a nutrition intervention. However, there were significant positive effects of SQ-LNSs on mean $z$ scores for growth, hemoglobin concentration, and indicators of iron status among both boys and girls. In addition, child sex did not modify 
the effect of SQ-LNSs on any indicators of child development. Thus, both boys and girls benefitted from the intervention.

Child birth order and maternal age both modified the effects of SQ-LNSs on more than 1 outcome domain. Effects of SQLNSs were greater among later-born children (i.e., those with at least 1 older sibling) than among first-born children with regard to stunting ( $13 \%$ vs. $9 \%$ relative reduction among later-born vs. first-born children, respectively), underweight (17\% vs. $6 \%$ reduction), low MUAC (23\% vs. $5 \%$ reduction), anemia (12 vs. 7 percentage point reduction), and several continuous outcomes (mean weight-for-age $z$ score, MUAC $z$ score, motor and fine motor scores, and hemoglobin and ferritin concentrations). Similarly, effects of SQ-LNSs were greater among children born to older mothers than among those born to younger mothers with regard to anemia ( $17 \%$ vs. $13 \%$ relative reduction) and mean motor and fine motor scores. Birth order and maternal age are positively correlated, so it is difficult to disentangle which factor is the most likely driver of these differences. Later-born children have at least 1 older sibling with whom they may compete for resources, making them potentially more vulnerable to malnutrition. In fact, we observed lower mean hemoglobin concentration and a greater prevalence of stunting and underweight among later-born (vs. first-born) children in the control groups in the IPD analysis, suggesting that they have a greater potential to benefit from nutritional supplementation.

Maternal education and depressive symptoms also appeared to modify the effect of SQ-LNSs on growth and development, but in seemingly disparate ways. Among children whose mothers had higher education or fewer depressive symptoms, there were greater effects of SQ-LNSs on growth. This may reflect a greater potential to respond to the intervention. Such mothers may have had greater autonomy and agency, and therefore may have been better able to adhere to advice regarding the recommended frequency or dosage of supplementation. By contrast, among children whose mothers had lower education, there were larger beneficial effects of SQ-LNSs on child development. Other studies have reported that maternal education is associated with developmental delays (59), and therefore these children may have lagged behind their peers and had greater room for improvement, with greater potential to benefit from an intervention.

Effect modification by season of assessment was significant for wasting and iron status, although the direction of the relation differed by outcome. Specifically, there were greater effects of SQ-LNSs on the prevalence of wasting among children assessed during the dry season (22\% reduction) compared with children assessed during the rainy season ( $8 \%$ reduction), which may reflect a greater potential to respond in the dry season due to a lower burden of infections. On the other hand, effects of SQ-LNSs on iron status were greater when it was measured during the rainy season, with an increase of $74 \%$ in ferritin concentration and a decrease of $63 \%$ in the prevalence of iron deficiency relative to the control groups. In the dry season, a benefit of SQ-LNSs was still apparent (44\% increase in ferritin and $47 \%$ reduction in the prevalence of iron deficiency), but the effect size was smaller. These results do not suggest that SQLNS interventions should be targeted to 1 season or another, but they have important implications with regard to interpreting results from evaluations, particularly if such studies have not been conducted longitudinally across a full calendar year. It is also important to note that the data included in the IPD meta-analysis were generally based on cross-sectional outcome assessments, which do not capture the multiple episodes of wasting/acute malnutrition, micronutrient deficiency, or anemia that may occur throughout the study period. In the PROMIS study in Mali, for example, the longitudinal prevalence of acute malnutrition was much higher than the cross-sectional prevalence, and the SQ-LNS intervention reduced the former by $29 \%$ but had no significant impact on the latter (35).

Finally, greater effects of SQ-LNSs on iron status and anemia were observed among children without inflammation at the time of assessment. This may reflect a greater potential to respond to a nutrition intervention among children without inflammation, who are likely to experience less inhibition of iron absorption or sequestration of circulating iron $(60,61)$. Despite this difference, significant positive effects of SQ-LNSs on these outcomes were apparent in both subgroups, with and without inflammation.

\section{Conclusions}

In summary, the evidence suggests that there are important benefits of SQ-LNSs for child survival, growth, anemia, iron status, and child development. It must be noted that the provision of SQ-LNSs is not a stand-alone intervention, as it should always be accompanied by messaging to reinforce IYCF recommendations, including a diverse diet with healthy foods from the key food groups. Nonetheless, it appears to play a protective role when access to nutrient-rich foods is limited for economic or other reasons. Of course, the benefits of SQ-LNSs must be weighed against the potential benefits of alternative interventions and the costs of delivering each type of intervention. At present, however, we are not aware of any other intervention for children that has a demonstrated impact in meta-analyses on all of the outcomes mentioned above.

The IPD meta-analysis included $>37,000$ children from a wide range of settings. For most outcomes, beneficial effects of SQ-LNSs were evident, regardless of region (Africa or South Asia), stunting burden, malaria prevalence, sanitation, water quality, duration of supplementation, frequency of contact, or average reported compliance with SQ-LNSs. Moreover, 6 of the 14 trials were conducted within existing community-based or clinic-based programs, so the evidence represents the continuum from efficacy to effectiveness trials. The sensitivity analyses demonstrated very similar results, indicating that the findings are robust. One limitation was that Bangladesh was the only country represented in the Southeast Asia region and Haiti was the only country represented in Latin America and the Caribbean, so additional data from countries outside of Africa would be valuable.

The effect modification results suggest that, for certain outcomes, targeting on the basis of population-level SES or burden of undernutrition may be worth considering, as the benefits of SQ-LNSs for iron status, anemia, and child development were larger in subgroups who had a greater potential to benefit from the intervention. The results also suggest that a greater impact of SQ-LNSs might be obtained by co-packaging them with interventions that alleviate constraints on response, such as the prevention and control of prenatal and child infections and inflammation; improving access to health care, including mental health care for women; and promoting early child development interventions that promote responsive caregiving. 
WASH interventions can help prevent infection and inflammation. Although trials that co-packaged a nutrition intervention (including SQ-LNSs) with household-level WASH interventions have not demonstrated greater impact on child growth outcomes compared with the nutrition intervention alone (31-34), it is possible that improvements in sanitation and water quality at the community level, not just at the individual household level, would yield a positive synergy between WASH and nutrition interventions (62-64). Other interventions to reduce infection or inflammation, such as deworming, provision of insecticidetreated bednets to reduce the risk of malaria, and other approaches to prevent childhood illness, were not tested directly in the above trials, so further research on effective co-interventions is needed.

The selection of intervention(s) for a given population should be based on the needs of the population, the goals of the program, as well as cost-to-benefit considerations. Although the latter is outside of the scope of this and the accompanying papers, work is underway to understand these economic dimensions. In the meantime, we recommend that policymakers and program planners consider including SQ-LNSs in the mix of interventions to reduce child mortality, stunting, wasting, anemia, iron deficiency, and delayed development. Whether to target SQ-LNSs to subgroups most in need, or to implement blanket distribution, is an important decision that depends on cost considerations and the key objectives of the program. If improved child development is the highest priority, our results indicate that targeting may be warranted, but for stunting, wasting, and anemia we found little evidence favoring targeting. The use of SQ-LNSs for the prevention of malnutrition is one of the few interventions that can help to achieve multiple Sustainable Development Goal targets simultaneously, as well as support the 3 pillars of the United Nations' 2016-2030 Global Strategy for Women's, Children's, and Adolescents' health (65): surviving (ending preventable deaths), thriving (ensuring health and well-being), and transforming (expanding enabling environments).

We thank all of the co-investigators, collaborators, study teams, participants, and local communities involved in the trials included in these analyses. These trials benefitted from the contributions of many partner organizations, including: icddr,b (JiVitA-4, Rang-Din Nutrition Study, and WASH Benefits trial in Bangladesh); the World Food Program (JiVitA-4 trial in Bangladesh); the Health District of Dandé and the relevant local health care authorities (iLiNS-ZINC trial in Burkina Faso); AfricSanté and Helen Keller International (PROMIS trials in Burkina Faso and Mali); Ministry of Public Health and Population (Haiti trial); Innovations for Poverty Action and the Kenya Medical Research Institute (WASH-Benefits trial in Kenya); Unité Programme National de Nutrition Communautaire, Government of Madagascar, and World Bank Health and Nutrition and Population Global Practice (MAHAY trial in Madagascar); the Ministry of Health and Child Care in Harare, Chirumanzu and Shurugwi districts, and Midlands Province (SHINE trial in Zimbabwe); the International Lipid-based Nutrient Supplements Project Steering Committee (iLiNS Project trials); and Nutriset (for development of SQ-LNSs). We thank Emily Smith for advice on IPD analysis methods.

The authors' responsibilities were as follows-KGD and CPS: drafted the manuscript with input from other coauthors; KGD: is responsible for final content; and all authors: contributed to and read and approved the final manuscript. KRW received a grant from Nutriset, SAS, outside of the submitted work during the period of this IPD analysis project. The other authors report no conflicts of interest.

\section{Data Availability}

Data described in the manuscript, code book, and analytic code will not be made available because they are compiled from 14 different trials, and access is under the control of the investigators of each of those trials.

\section{Sponsorship Acknowledgment}

This supplement is sponsored by the Institute for Global Nutrition at the University of California, Davis, with funding from the Bill \& Melinda Gates Foundation to UC Davis via grant OPP49817.

\section{References}

1. Black RE, Victora CG, Walker SP, Bhutta ZA, Christian P, de Onis M, Ezzati M, Grantham-McGregor S, Katz J, Martorell R, et al. Maternal and child undernutrition and overweight in low-income and middleincome countries. Lancet North Am Ed 2013;382:427-51.

2. UNICEF; World Health Organization; International Bank for Reconstruction and Development/The World Bank. Levels and trends in child malnutrition: key findings of the 2020 edition of the Joint Child Malnutrition Estimates. Geneva (Switzerland): World Health Organization; 2020.

3. Black MM, Walker SP, Fernald LCH, Andersen CT, DiGirolamo AM, Lu C, McCoy DC, Fink G, Shawar YR, Shiffman J, et al. Early childhood development coming of age: science through the life course. Lancet North Am Ed 2017;389:77-90.

4. Bailey RL, West KP Jr., Black RE. The epidemiology of global micronutrient deficiencies. Ann Nutr Metab 2015;66(Suppl 2):22-33.

5. UNICEF. Improving child nutrition: the achievable imperative for global progress [Internet]. New York: UNICEF; 2013. Available from: https://www.unicef.org/publications/index_68661.html(accessed 25 September 2020).

6. Barks A, Hall AM, Tran PV, Georgieff MK. Iron as a model nutrient for understanding the nutritional origins of neuropsychiatric disease. Pediatr Res 2019;85:176-82.

7. Grantham-McGregor S, Cheung YB, Cueto S, Glewwe P, Richter L, Strupp B; International Child Development Steering Group. Developmental potential in the first 5 years for children in developing countries. Lancet North Am Ed 2007;369:60-70.

8. Stewart CP, Iannotti L, Dewey KG, Michaelsen KF, Onyango AW. Contextualising complementary feeding in a broader framework for stunting prevention. Matern Child Nutr 2013;9(Suppl 2):27-45.

9. Prendergast AJ, Humphrey JH. The stunting syndrome in developing countries. Paediatr Int Child Health 2014;34:250-65.

10. Millward DJ. Nutrition, infection and stunting: the roles of deficiencies of individual nutrients and foods, and of inflammation, as determinants of reduced linear growth of children. Nutr Res Rev 2017;30:50-72.

11. Harper KM, Mutasa M, Prendergast AJ, Humphrey J, Manges AR. Environmental enteric dysfunction pathways and child stunting: a systematic review. PLoS Negl Trop Dis 2018;12:e0006205.

12. Schoenbuchner SM, Dolan C, Mwangome M, Hall A, Richard SA, Wells JC, Khara T, Sonko B, Prentice AM, Moore SE. The relationship between wasting and stunting: a retrospective cohort analysis of longitudinal data in Gambian children from 1976 to 2016. Am J Clin Nutr 2019;110:498-507.

13. Black RE. Patterns of growth in early childhood and infectious disease and nutritional determinants. Nestle Nutr Inst Workshop Ser 2017;87:63-72.

14. Dewey KG. Reducing stunting by improving maternal, infant and young child nutrition in regions such as South Asia: evidence, challenges and opportunities. Matern Child Nutr 2016;12(Suppl 1):27-38.

15. PAHO/WHO (Pan American Health Organization/World Health Organization). Guiding principles for complementary feeding of the breastfed child. Washington (DC): PAHO; 2003.

16. UNICEF. Improving young children's diets during the complementary feeding period. UNICEF Programming Guidance. New York: UNICEF; 2020 . 
17. Headey DD, Alderman HH. The relative caloric prices of healthy and unhealthy foods differ systematically across income levels and continents. J Nutr 2019;149:2020-33.

18. Miller V, Yusuf S, Chow CK, Dehghan M, Corsi DJ, Lock K, Popkin B, Rangarajan S, Khatib R, Lear SA, et al. Availability, affordability, and consumption of fruits and vegetables in 18 countries across income levels: findings from the Prospective Urban Rural Epidemiology (PURE) study. Lancet Glob Health 2016;4:e695-703.

19. Dewey KG, Vitta BS. Strategies for ensuring adequate nutrient intake for infants and young children during the period of complementary feeding. Insight Issue No. 7, p. 14. Alive \& Thrive Technical Brief: Washington, DC: Alive \& Thrive; 2013 [Internet]. Available from: https://www.aliveandthrive.org/en/resources/ensuring - adequate-nutrient-intake-during-the-period-of-complementary-feedi ng-insight-series (accessed Aug 192021 ).

20. Das JK, Hoodbhoy Z, Salam RA, Bhutta AZ, Valenzuela-Rubio NG, Weise Prinzo Z, Bhutta ZA. Lipid-based nutrient supplements for maternal, birth, and infant developmental outcomes. Cochrane Database Syst Rev 2018;8:CD012610.

21. Das JK, Salam RA, Hadi YB, Sadiq Sheikh S, Bhutta AZ, Weise Prinzo Z, Bhutta ZA. Preventive lipid-based nutrient supplements given with complementary foods to infants and young children 6 to 23 months of age for health, nutrition, and developmental outcomes. Cochrane Database Syst Rev 2019;5:CD012611.

22. Stewart CP, Wessells KR, Arnold CD, Huybregts L, Ashorn P, Becquey E, Humphrey JH, Dewey KG. Lipid-based nutrient supplements and all-cause mortality in children 6-24 months of age: a meta-analysis of randomized controlled trials. Am J Clin Nutr 2020;111:207-18.

23. Dewey KG, Wessells KR, Arnold CD, Prado EL, Abbeddou S, Adu-Afarwuah S, Ali H, Arnold BF, Ashorn P, Ashorn U, et al. Characteristics that modify the effect of small-quantity lipid-based nutrient supplementation on child growth: an individual participant data meta-analysis of randomized controlled trials. Am J Clin Nutr 2021;114(Suppl 11):15S-42S.

24. Prado EL, Arnold CD, Wessells KR, Stewart CP, Abbeddou S, AduAfarwuah S, Arnold BF, Ashorn U, Ashorn P, Becquey E, et al. Smallquantity lipid-based nutrient supplements for children age 6-24 months: a systematic review and individual participant data meta-analysis of effects on developmental outcomes and effect modifiers. Am J Clin Nutr2021;114(Suppl 11):43S-67S

25. Wessells K, Arnold C, Stewart C, Prado E, Abbeddou S, AduAfarwuah S, Arnold BF, Ashorn P, Ashorn U, Becquey E, et al. Characteristics that modify the effect of small-quantity lipid-based nutrient supplementation on child anemia and micronutrient status: an individual participant data meta-analysis of randomized controlled trials. Am J Clin Nutr2021;114(Suppl 11):68S-94S

26. Arimond $\mathrm{M}$, Zeilani $\mathrm{M}$, Jungjohann $\mathrm{S}$, Brown $\mathrm{KH}$, Ashorn $\mathrm{P}$, Allen LH, Dewey KG. Considerations in developing lipid-based nutrient supplements for prevention of undernutrition: experience from the International Lipid-Based Nutrient Supplements (iLiNS) Project. Matern Child Nutr 2015;11(Suppl 4):31-61.

27. Hemsworth J, Kumwenda C, Arimond M, Maleta K, Phuka J, Rehman AM, Vosti SA, Ashorn U, Filteau S, Dewey KG, et al. Lipid-based nutrient supplements increase energy and macronutrient intakes from complementary food among Malawian infants. J Nutr 2016;146:32634.

28. Home Fortification Technical Advisory Group. Home page[Internet]. Available from: http://www.hftag.org (accessed 1 July 2020).

29. Adu-Afarwuah S, Lartey A, Brown KH, Zlotkin S, Briend A, Dewey KG. Randomized comparison of 3 types of micronutrient supplements for home fortification of complementary foods in Ghana: effects on growth and motor development. Am J Clin Nutr 2007;86:412-20.

30. Phuka JC, Maleta K, Thakwalakwa C, Cheung YB, Briend A, Manary MJ, Ashorn P. Complementary feeding with fortified spread and incidence of severe stunting in 6- to 18-month-old rural Malawians. Arch Pediatr Adolesc Med 2008;162:619-26.

31. Luby SP, Rahman M, Arnold BF, Unicomb L, Ashraf S, Winch PJ, Stewart CP, Begum F, Hussain F, Benjamin-Chung J, et al. Effects of water quality, sanitation, handwashing, and nutritional interventions on diarrhoea and child growth in rural Bangladesh: a cluster randomised controlled trial. Lancet Glob Health 2018;6:e302-e15.

32. Null C, Stewart CP, Pickering AJ, Dentz HN, Arnold BF, Arnold CD, Benjamin-Chung J, Clasen T, Dewey KG, Fernald LCH, et al. Effects of water quality, sanitation, handwashing, and nutritional interventions on diarrhoea and child growth in rural Kenya: a cluster-randomised controlled trial. Lancet Glob Health 2018;6:e316-e29.

33. Humphrey JH, Mbuya MNN, Ntozini R, Moulton LH, Stoltzfus RJ, Tavengwa NV, Mutasa K, Majo F, Mutasa B, Mangwadu G, et al Independent and combined effects of improved water, sanitation, and hygiene, and improved complementary feeding, on child stunting and anaemia in rural Zimbabwe: a cluster-randomised trial. Lancet Glob Health 2019;7:e132-47.

34. Prendergast AJ, Chasekwa B, Evans C, Mutasa K, Mbuya MNN, Stoltzfus RJ, Smith LE, Majo FD, Tavengwa NV, Mutasa B, et al. Independent and combined effects of improved water, sanitation, and hygiene, and improved complementary feeding, on stunting and anaemia among HIV-exposed children in rural Zimbabwe: a clusterrandomised controlled trial. Lancet Child Adolesc Health 2019;3:77_ 90.

35. Huybregts L, Le Port A, Becquey E, Zongrone A, Barba FM, Rawat R, Leroy JL, Ruel MT. Impact on child acute malnutrition of integrating small-quantity lipid-based nutrient supplements into community-level screening for acute malnutrition: a cluster-randomized controlled trial in Mali. PLoS Med 2019;16(8):e1002892.

36. Becquey E, Huybregts L, Zongrone A, Le Port A, Leroy JL, Rawat R, Toure M, Ruel MT. Impact on child acute malnutrition of integrating a preventive nutrition package into facility-based screening for acute malnutrition during well-baby consultation: a cluster-randomized controlled trial in Burkina Faso. PLoS Med 2019;16(8):e1002877.

37. Wessells KR, Stewart C, Arnold CD, Dewey K, Prado E. Modifiers of the effect of LNS provided to infants and children 6 to 24 months of age on growth, anemia, micronutrient status and development outcomes [Internet]. Open Science Framework. Available from: https://osf.io/yms fu.

38. Wessells K, Dewey K, Stewart C, Arnold C, Prado E. Modifiers of the effect of LNS provided to infants and children 6 to 24 months of age on growth outcomes: a systematic review and meta-analysis of individual participant data from randomized controlled trials in low-income and middle-income countries [Internet]. PROSPERO 2019 CRD42019146592 Available from: https://www.crd.york.ac.uk/prosper o/display_record.php?ID=CRD42019146592.

39. Wessells K, Prado E, Dewey K, Stewart C, C A. Modifiers of the effect of LNS provided to infants and children 6 to 24 months of age on developmental outcomes: a systematic review and meta-analysis of individual participant data from randomized controlled trials in low-income and middle-income countries [Internet]. PROSPERO 2020 CRD42020159971 Available from: https://www.crd.york.ac.uk/prosper o/display record.php?ID=CRD42020159971.

40. Wessells K, Dewey K, Stewart C, Arnold C, Prado E. Modifiers of the effect of LNS provided to infants and children 6 to 24 months of age on anemia and MN status outcomes: a systematic review and metaanalysis of individual participant data from randomized controlled trials in low-income and middle-income countries [Internet]. PROSPERO 2020 CRD42020156663 Available from: https://www.crd.york.ac.uk/p rospero/display_record.php?ID=CRD42020156663.

41. Christian P, Shaikh S, Shamim AA, Mehra S, Wu L, Mitra M, Ali H, Merrill RD, Choudhury N, Parveen M, et al. Effect of fortified complementary food supplementation on child growth in rural Bangladesh: a cluster-randomized trial. Int J Epidemiol 2015;44:186276.

42. Dewey KG, Mridha MK, Matias SL, Arnold CD, Cummins JR, Khan MS, Maalouf-Manasseh Z, Siddiqui Z, Ullah MB, Vosti SA. Lipidbased nutrient supplementation in the first $1000 \mathrm{~d}$ improves child growth in Bangladesh: a cluster-randomized effectiveness trial. Am J Clin Nutr 2017; 105:944-57

43. Hess SY, Abbeddou S, Jimenez EY, Some JW, Vosti SA, Ouedraogo ZP, Guissou RM, Ouedraogo JB, Brown KH. Small-quantity lipidbased nutrient supplements, regardless of their zinc content, increase growth and reduce the prevalence of stunting and wasting in young Burkinabe children: a cluster-randomized trial. PLoS One 2015; 10:e0122242.

44. Adu-Afarwuah S, Lartey A, Okronipa H, Ashorn P, Peerson JM, Arimond M, Ashorn U, Zeilani M, Vosti S, Dewey KG. Smallquantity, lipid-based nutrient supplements provided to women during pregnancy and 6 mo postpartum and to their infants from 6 mo of age increase the mean attained length of 18-mo-old children in semiurban Ghana: a randomized controlled trial. Am J Clin Nutr 2016;104: 797-808. 
45. Iannotti LL, Dulience SJ, Green J, Joseph S, Francois J, Antenor ML, Lesorogol C, Mounce J, Nickerson NM. Linear growth increased in young children in an urban slum of Haiti: a randomized controlled trial of a lipid-based nutrient supplement. Am J Clin Nutr 2014;99:198-208.

46. Galasso E, Weber AM, Stewart CP, Ratsifandrihamanana L, Fernald LCH. Effects of nutritional supplementation and home visiting on growth and development in young children in Madagascar: a cluster-randomised controlled trial. Lancet Glob Health 2019;7: e1257-e68.

47. Ashorn P, Alho L, Ashorn U, Cheung YB, Dewey KG, Gondwe A, Harjunmaa U, Lartey A, Phiri N, Phiri TE, et al. Supplementation of maternal diets during pregnancy and for 6 months postpartum and infant diets thereafter with small-quantity lipid-based nutrient supplements does not promote child growth by 18 months of age in rural Malawi: a randomized controlled trial. J Nutr 2015;145:1345-53.

48. Maleta KM, Phuka J, Alho L, Cheung YB, Dewey KG, Ashorn U, Phiri N, Phiri TE, Vosti SA, Zeilani M, et al. Provision of 10-40 g/d lipidbased nutrient supplements from 6 to 18 months of age does not prevent linear growth faltering in Malawi. J Nutr 2015;145:1909-15.

49. Smuts CM, Matsungo TM, Malan L, Kruger HS, Rothman M, Kvalsvig JD, Covic N, Joosten K, Osendarp SJM, Bruins MJ, et al. Effect of small-quantity lipid-based nutrient supplements on growth, psychomotor development, iron status, and morbidity among 6- to 12mo-old infants in South Africa: a randomized controlled trial. Am J Clin Nutr 2019;109:55-68.

50. Tam E, Keats EC, Rind F, Das JK, Bhutta AZA. Micronutrient supplementation and fortification interventions on health and development outcomes among children under-five in low- and middle-income countries: a systematic review and meta-analysis. Nutrients 2020;12:289.

51. Arikpo D, Edet ES, Chibuzor MT, Odey F, Caldwell DM. Educational interventions for improving primary caregiver complementary feeding practices for children aged 24 months and under. Cochrane Database Syst Rev 2018;5:CD011768.

52. Matsuyama M, Harb T, David M, Davies PS, Hill RJ. Effect of fortified milk on growth and nutritional status in young children: a systematic review and meta-analysis. Public Health Nutr 2017;20: 1214-25.

53. Sguassero Y, de Onis M, Bonotti AM, Carroli G. Community-based supplementary feeding for promoting the growth of children under five years of age in low and middle income countries. Cochrane Database Syst Rev 2012;6:CD005039.

54. Eichler K, Wieser S, Ruthemann I, Brugger U. Effects of micronutrient fortified milk and cereal food for infants and children: a systematic review. BMC Public Health 2012;12:506.

55. Suchdev PS, Jefferds MED, Ota E, da Silva Lopes K, De-Regil LM. Home fortification of foods with multiple micronutrient powders for health and nutrition in children under two years of age. Cochrane Database Syst Rev 2020;2:CD008959.
56. Prado EL, Larson LM, Cox K, Bettencourt K, Kubes JN, Shankar AH. Do effects of early life interventions on linear growth correspond to effects on neurobehavioural development? A systematic review and meta-analysis. Lancet Glob Health 2019;7: e1398-e413.

57. Aboud FE, Yousafzai AK. Global health and development in early childhood. Annu Rev Psychol 2015;66:433-57.

58. Wells JC. Natural selection and sex differences in morbidity and mortality in early life. J Theor Biol 2000;202:65-76.

59. Wachs TD, Rahman A. The nature and impact of risk and protective influences on children's development in low-income countries. In: Britto PR, Engle PL, Super CM, editors. Handbook of early childhood development research and its impact on global policy. New York: Oxford University Press; 2013. p. 85-122.

60. Nemeth E, Ganz T. The role of hepcidin in iron metabolism. Acta Haematol 2009;122:78-86.

61. Hurrell RF. Influence of inflammatory disorders and infection on iron absorption and efficacy of iron-fortified foods. Nestle Nutr Inst Workshop Ser 2012;70:107-16.

62. Cumming O, Arnold BF, Ban R, Clasen T, Esteves Mills J, Freeman MC, Gordon B, Guiteras R, Howard G, Hunter PR, et al. The implications of three major new trials for the effect of water, sanitation and hygiene on childhood diarrhea and stunting: a consensus statement. BMC Med 2019;17:173.

63. Husseini M, Darboe MK, Moore SE, Nabwera HM, Prentice AM Thresholds of socio-economic and environmental conditions necessary to escape from childhood malnutrition: a natural experiment in rural Gambia. BMC Med 2018;16:199.

64. Pickering AJ, Null C, Winch PJ, Mangwadu G, Arnold BF, Prendergast AJ, Njenga SM, Rahman M, Ntozini R, Benjamin-Chung J, et al. The WASH Benefits and SHINE trials: interpretation of WASH intervention effects on linear growth and diarrhoea. Lancet Glob Health 2019;7:e1139-46.

65. Every Woman Every Child. The global strategy for women's, children's and adolescent's health (2016-2030) [Internet]. 2015. Available from: http://www.globalstrategy.everywomaneverychild.org(accessed 26 January 2021).

66. World Health Organization. World malaria report 2018 [Internet]. Geneva (Switzerland): World Health Organization; 2018. Available from: https://www.who.int/malaria/publications/world-malaria-report2018/report/en/(accessed 26 August 2019).

67. WHO, UNICEF Joint Monitoring Programme. Drinking water [Internet]. [cited 26 August, 2019]. Geneva, Switzerland: World Health Organization; 2017. Licence: CC BY-NC-SA 3.0 IGO. Available from: http://washdata.org/monitoring/drinking-water.

68. WHO, UNICEF Joint Monitoring Programme. Sanitation [Internet]. [cited 26 August, 2019]. Geneva, Switzerland: World Health Organization; 2017. Licence: CC BY-NC-SA 3.0 IGO. Available from: http://washdata.org/monitoring/sanitation. 